des Sowjetkommunismus beschäftigte sich Kohn in dem Band The Twentieth Century. A Mid-way Account of the Western World. ${ }^{400}$

In den Vereinigten Staaten unterstützte Kohn im Anschluss an das Ende des Zweiten Weltkrieges - neben Martin Buber, Erich Fromm, James Marshall, Maurice Hexter, David Riesman, Hannah Arendt und dem Herausgeber der Zeitschrift Commentary, Elliot Cohen - die jüdische Organisation Ihud von Judah Magnes und engagierte sich politisch in Bezug auf die Gründung des Staates Israel. In diesem Kontext befürwortete er eine arabisch-israelische, d. h. binationale Lösung des Palästinaproblems.

Neben den bereits angeführten diversen Veröffentlichungen trat Kohn in den 1950er- und 1960er-Jahren zudem als Autor von zahlreichen weiteren Büchern in Erscheinung. ${ }^{401} \mathrm{Zu}$ seinen Freunden in den Vereinigten Staaten gehörte unter anderem der Totalitarismusforscher Sigmund Neumann. ${ }^{402}$ Zuletzt lebte Kohn in Philadelphia, wo er am 16. März 1971 verstarb.

\title{
4.8 Hannah Arendt
}

Die Schriften Hannah Arendts wurden fast sämtlich unter dem Eindruck der übermächtigen Destruktionsgeschichte des 20. Jahrhunderts angefertigt. Aufgrund der existenziellen Erfahrung von Verfolgung, des Flüchtlings, der Asylsuchenden, Internierten und Staatenlosen, von Gewalt und Terror - sprich einer paradigmatischen Grunderfahrung des letzten Jahrhunderts - kam es bei ihr ausdrücklich zu einer Verflechtung von Werk und Biografie. Die mit ihrer 1951 in den Vereinigten Staaten erschienenen Untersuchung The Origins of Totalitarianism ${ }^{403}$ international bekannt gewordene Arendt gehörte frühzeitig zu den Autorinnen des Monat. ${ }^{404}$ Neben einem literarischen Porträt Hermann Brochs ${ }^{405}$ und einem Rezensionsaufsatz zur Geschichte des >Dritten Reiches` mit dem Titel Bei Hitler zu Tisch ${ }^{406}$ standen drei Auszüge ihrer

400 Ders., The Twentieth Century. A Mid-way Account of the Western World, New York 1949 (dt. Ausg.: Das Zwanzigste Jahrhundert. Eine Zwischenbilanz des Westens, Zürich/Wien/Konstanz 1950); siehe hierzu Joachim G. Leithäuser, Unbarmherzige Ratio, in: Der Monat 3 (1951), H. 30, S. 655-657 (Rezension). Siehe hierzu auch Kap. II.1.

401 Siehe exemplarisch Hans Kohn, Is the liberal west in decline, London 1957 (dt. Ausg.: Ist die freie Welt zum Untergang verurteilt?, Köln 1959).

402 Neumann, Permanent Revolution. Kohn zeichnete für die nach Neumanns Tod im Jahre 1962 herausgegebene zweite Auflage verantwortlich. Diesen Hinweis entnahm ich dem Aufsatz von Söllner, Sigmund Neumanns »Permanent Revolution«, S. 53, Anm. 2.

403 Arendt, The Origins of Totalitarianism. Zu den aus Anlass des fünfzigsten Erscheinungsjahres der Studie veranstalteten Tagungen an der Berlin-Brandenburgischen Akademie der Wissenschaft in Berlin und an der Carl von Ossietzky Universität Oldenburg im Dezember 2001 siehe Antonia Crunenberg (Hg.), Totalitäre Herrschaft und republikanische Demokratie. Fünfzig Jahre The Origins of Totalitarianism von Hannah Arendt, Frankfurt a. M. 2003.

404 Vor der Mitarbeit für den Monat veröffentlichte Arendt - wie auch beispielsweise Kohn - bereits in der von ihren Freunden Dolf Sternberger und Karl Jaspers ab 1945 (bis 1949) (mit-)herausgegebenen Zeitschrift Die Wandlung. Sie publizierte dort die Beiträge Franz Kafka, von neuem gewürdigt, Organisierte Schuld, Über den Imperialismus, Konzentrationsläger, Es gibt nur ein einziges Menschenrecht sowie Parteien und Bewegungen.

405 Hermann Broch und der moderne Roman, in: Der Monat 1 (1949), H. 8/9, S. 147-151.

406 Bei Hitler zu Tisch, in: Der Monat 4 (1951), H. 37, S. 85-90 (Rezension). 
Totalitarismusstudie im Mittelpunkt ihrer Publikationen. ${ }^{407}$ Danach folgten die Beiträge Was ist Autorität $t^{408}$, Krise der Erziehung ${ }^{409}$ und Rosa Luxemburg ${ }^{410}$.

Arendt wurde am 14. Oktober 1906 in Lingen bei Hannover als einzige Tochter von assimilierten jüdischen Eltern aus dem Bildungsbürgertum geboren. ${ }^{411}$ Die Eltern stammten aus Königsberg und waren in der Jugend in der sozialdemokratischen Bewegung engagiert und sympathisierten mit den Ideen der Jugendbewegung. Noch bevor der Vater, ein Ingenieur, frühzeitig verstarb, übersiedelte die Familie nach Königsberg. Arendt besuchte dort ein Mädchengymnasium, zeigte großes Interesse für Philosophie und las im Alter von 14 Jahren unter anderem Kants Kritik der reinen Vernunft und Jaspers Zur Psychologie der Weltanschauungen. Nach dem Abitur begann sie 1924 in Marburg bei Martin Heidegger Philosophie und bei Rudolf Bultmann Theologie und Griechisch zu studieren. 1925 setzte sie ihr Studium bei Edmund Husserl in Freiburg fort und promovierte 1928 in Heidelberg bei Karl Jaspers über den Liebesbegriff bei Augustinus. ${ }^{412}$

1929 heiratete Arendt den Philosophen Günther Stern - den später unter seinem Pseudonym Günther Anders bekanntgewordenen Technik- und Zivilisationskritiker und begann ein Jahr danach mit der Arbeit über die jüdische Romantikerin Rahel Varnhagen, die sie 1938 im Exil beendete. ${ }^{413}$ Nach der Ernennung Hitlers zum Reichskanzler wurde Arendt im Sommer 1933 von der Gestapo verhaftet und infolge glücklicher Umstände nach wenigen Tagen wieder entlassen. ${ }^{414}$ Ein lebensgeschichtlicher Schock war für Arendt das Verhalten von befreundeten Intellektuellen, ${ }^{415}$ genauer die Gleichschaltung von Freunden, denen zu Hitler »sehr viel einfiel«. Denn, so Arendt:

Das persönliche Problem war doch nicht etwa, was unsere Feinde taten, sondern was unsere Freunde taten. Was damals in der Welle von Gleichschaltung, die ja ziemlich

407 Hierbei handelte es sich um die Auszüge Der imperialistische Charakter, Totalitäre Propaganda sowie Die Geheimpolizei. Siehe zu Arendt auch die entsprechenden Ausführungen im Einleitungskapitel sowie Kap. II.1.

408 Was ist Autorität?, in: Der Monat 8 (1956), H. 89, S. 29-44 (hierbei handelte es sich um einen von Arendt auf der internationalen Tagung des »Kongresses für die Freiheit der Kultur«vom 12. bis 17. September 1955 in Mailand gehaltenen Vortrag).

409 Die Krise der Erziehung. Gedanken zur »Progressive Education«, in: Der Monat 11 (1959), H. 124, S. 48-61.

410 Rosa Luxemburg, in: Der Monat 20 (1968), H. 243, S. 28-40.

411 Im Folgenden beziehe ich mich im Wesentlichen auf die Bücher: Seyla Benhabib, Hannah Arendt. Die melancholische Denkerin der Moderne, Hamburg 1998; Wolfgang Heuer, Hannah Arendt. Mit Selbstzeugnissen und Bilddokumenten, Reinbek bei Hamburg 1987; ders., Citizen. Persönliche Integrität und politisches Handeln. Eine Rekonstruktion des politischen Humanismus Hannah Arendts, Berlin 1992; Young-Bruehl, Hannah Arendt. Leben, Werk und Zeit sowie Friedrich Georg Friedmann, Hannah Arendt - Eine deutsche Jüdin im Zeitalter des Totalitarismus, München/Zürich 1985.

412 Der Liebesbegriff bei Augustin. Versuch einer philosophischen Interpretation, Berlin 1929.

413 Rahel Varnhagen, The Life of a Jewess, London 1958 (dt. Ausg.: Rahel Varnhagen, Lebensgeschichte einer deutschen Jüdin aus der Romantik, München 1959).

414 Siehe zu den genauen Umständen Arendt, Was bleibt?, S. $14 \mathrm{f}$.

415 Diese Erfahrung bezog sich in allererster Linie auf das Verhalten von Martin Heidegger und von ihrem Studienfreund, dem nach dem Zweiten Weltkrieg bekannt gewordenen Germanisten Benno von Wiese. 
freiwillig war, jedenfalls noch nicht unter dem Druck des Terrors vorging: das war, als ob sich ein leerer Raum um einen bildete. Ich lebte in einem intellektuellen Milieu, ich kannte aber auch andere Menschen. Und ich konnte feststellen, dass unter den Intellektuellen die Gleichschaltung sozusagen die Regel war. Aber unter den andern nicht. Und das hab ich nie vergessen. ${ }^{416}$

Danach flüchtete Arendt über Prag nach Paris, wo sie sich der zionistischen Bewegung anschloss und sich als Generalsekretärin der französischen Abteilung der Jugend-Alija mehrere Jahre um die Auswanderung jüdischer Flüchtlingskinder nach Palästina bemühte. Durch ihren Freund Walter Benjamin ${ }^{417}$ lernte sie den ehemaligen Kommunisten Heinrich Blücher kennen, den sie, nach der Scheidung von Günther Stern, heiratete. 1937 wurde ihr die deutsche Staatsangehörigkeit aberkannt. Nach der Eroberung Frankreichs durch die Wehrmacht wurde Arendt im Mai 1940 in dem südfranzösischen Konzentrationslager Gurs interniert. Nach fünf Wochen wurde sie wieder freigelassen. »Wenig später war es kaum noch möglich, das Lager zu verlassen; die Insassen wurden 1943 in die deutschen Vernichtungslager deportiert. « ${ }^{418}$ Arendt flüchtete mit ihrem Mann und ihrer Mutter über Spanien nach Portugal und konnte von dort aus im Mai 1941 nach New York ausreisen.

In New York veröffentlichte Arendt in der Zeitschrift Jewish Social Studies und schrieb in der deutsch-jüdischen Emigrantenzeitung Aufbau regelmäßig Kolumnen. ${ }^{419}$ Dort erfuhr sie von Auschwitz, d.h. der nationalsozialistischen »Fabrikation der Leichen«. Wie gezeigt, wurde der Holocaust zur eigentlichen Schockerfahrung für Arendt. ${ }^{420}$ Im Schocken Verlag arbeitete sie als Lektorin und schloss sich der jüdischen Organisation Ihud von Judah Magnes an - die unter anderem auch von Martin Buber, Erich Fromm, David Riesman, Hans Kohn und dem Herausgeber der amerikanischen Zeitschrift Commentary, Elliot Cohen, unterstützt wurde - und warnte vor einer Gründung des Staates Israels qua Gewaltakt und sprach sich für eine binationale Lösung des Palästinaproblems aus. ${ }^{421}$

416 Arendt, Was bleibt?, S. 20.

417 Der Philosoph, Gesellschafts- und Literaturkritiker sowie Mitarbeiter des Frankfurter Institutes für Sozialforschung, Walter Benjamin übergab zum Zeitpunkt der akuten Bedrohung Arendts die unter dem Eindruck der historischen Katastrophe geschriebenen Thesen über Geschichte. Aus Angst vor der Verhaftung durch die Gestapo beendete Benjamin auf der Flucht im September 1940 in Port Bou an der französisch-spanischen Grenze sein Leben durch eigene Hand. Vgl. Hannah Arendt, Walter Benjamin, in: Dies., Menschen in finsteren Zeiten. Herausgegeben von Ursula Ludz, 2. Aufl., München 1989, S. 185-242 (amerik. Ausgabe: Men in Dark Times, New York 1969).

418 Heuer, Hannah Arendt, S. 36.

419 Siehe Hannah Arendt, Vor Antisemitismus ist man nur noch auf dem Monde sicher. Beiträge für die deutsch-jüdische Emigrantenzeitung Aufbau 1941-1945. Hg. von Marie Luise Knott, München 2000.

420 Siehe die einleitenden Ausführungen in Kap. II.2; vgl. auch Hannah Arendt, Die Vollendete Sinnlosigkeit, in: Dies., Nach Auschwitz. Essays \& Kommentare 1, Berlin 1989, S. 7-30.

421 Siehe bes. Hannah Arendt, Frieden oder Waffenstillstand im Nahen Osten?, in: Dies., Israel, Palästina und der Antisemitismus, Berlin 1991, S. 39-75. Vgl. Hannah Arendt, Die Krise des Zionismus. Essays \& Kommentare 2. Herausgegeben von Eike Geisel und Klaus Bittermann. Mit einem Nachwort von Henryk M. Broder, Berlin 1989. 
Arendt veröffentlichte - wie erwähnt - in der nach Kriegsende gegründeten deutschen Zeitschrift Die Wandlung mehrere Beiträge, darunter Organisierte Schuld ${ }^{422}$ sowie Über den Imperialismus ${ }^{423}$, Konzentrationsläger ${ }^{424}$, Es gibt nur ein einziges Menschenrecht ${ }^{425}$ sowie Parteien und Bewegungen ${ }^{426}$, die Vorfassungen von fast wortgetreuen Kapiteln von The Origins of Totalitarianism darstellten. Als Geschäftsführerin der Jewish Cultural Reconstruction Incorporated reiste sie 1949 zum ersten Mal wieder nach Deutschland, um zur Rettung jüdischer Kulturgüter beizutragen. ${ }^{427}$ Den bis März 1950 dauernden Aufenthalt nutzte sie auch, um den Nachwirkungen des Naziregimes nachzuspüren. Bereits in einem Brief am 14. Dezember an ihren Ehemann schilderte sie ihre deprimierenden Erlebnisse:

Weißt Du eigentlich, wie recht Du hattest, nie wieder zurück zu wollen? Die Sentimentalität bleibt einem im Halse stecken, nachdem sie einem erst in die Kehle gestiegen ist. Die Deutschen leben von der Lebenslüge und der Dummheit. Letztere stinkt zum Himmel. Wenn du hier eine Woche lang sämtliche Zeitungen von rechts bis links gelesen hast, dann bist du reif für die Rückfahrt. [...] Nicht wahr ist, daß es hier viele Nazis gibt. Sie sehnen sich halt nach Hitler ohne Krieg zurück, verstehen überhaupt nichts - die Studenten so wenig wie die Arbeiter. ${ }^{428}$

In einem zuerst im Herbst 1950 in der US-amerikanischen Zeitschrift Commentary erschienenen Bericht über ihren Deutschlandbesuch akzentuierte sie ihre Eindrücke.

Der wohl hervorstechendste und auch erschreckendste Aspekt der deutschen Realitätsflucht«, so Arendt, »liegt jedoch in der Haltung, mit Tatsachen so umzugehen, als handele es sich um bloße Meinungen. Beispielsweise kommt als Antwort auf die Frage, wer den Krieg begonnen habe - ein keineswegs heiß umstrittenes Thema - eine überraschende Vielfalt von Meinungen zutage. ${ }^{429}$

Vor dem Hintergrund, dass selbst mit unbestreitbaren Fakten des >Dritten Reiches «o umgegangen wurde, als handele es sich um bloße Meinungen, stellte es für Arendt eine gängige Praxis der Deutschen dar, auch der Wirklichkeit der NS-Verbrechen aus dem Wege zu gehen: »Aus der Wirklichkeit der Todesfabriken wird eine bloße Möglichkeit: die Deutschen hätten nur das getan, wozu andere auch fähig seien (was natürlich mit vielen Beispielen illustriert wird) oder wozu andere künftig in der Lage wären; deshalb wird jeder, der dieses Thema anschneidet, ipso facto der Selbstgerechtigkeit verdächtigt. « ${ }^{430}$ Insofern korrespondierte die Flucht vor der Wirklichkeit mit der Flucht

422 Organisierte Schuld, in: Die Wandlung 1 (1946), H. 4, S. 333-344.

423 Über den Imperialismus, in: Die Wandlung 1 (1946), H. 8, S. 650-666.

424 Konzentrationsläger, in: Die Wandlung 3 (1948), H. 4, S. 309-330.

425 Es gibt nur ein einziges Menschenrecht, in: Die Wandlung 4 (1949), o. Nr. (Herbstheft), S. 754-770.

426 Parteien und Bewegungen, in: Die Wandlung 4 (1949), H. 6, S. 459-473.

427 Zu den Eindrücken, Erfahrungen und Erkenntnissen der von August 1949 bis März 1950 dauernden Reise siehe Arendt, Besuch in Deutschland.

428 Hannah Arendt/Heinrich Blücher, Briefe 1936-1968, München/Zürich 1996, S. 175.

429 Arendt, Besuch in Deutschland, S. 29.

430 Ebd., S. $27 \mathrm{f}$. 
vor der Verantwortung, die das »Hitlerregime« den Deutschen aufbürdete. Überhaupt geschah für Arendt der "gravierendste Irrtum der amerikanischen Entnazifizierungspolitik« bereits ganz am Anfang, »als nämlich versucht wurde, das Gewissen des deutschen Volkes angesichts der Ungeheuerlichkeit der in seinem Namen und unter Bedingungen organisierter Komplizenschaft begangener Verbrechen wachzurütteln «. ${ }^{431}$

Nach der Veröffentlichung ihrer Totalitarismusstudie und der bereits angesprochenen Kritik, dass es nämlich in ihrem Buch ein Ungleichgewicht im Hinblick auf die Analyse der beiden totalitären Herrschaftssysteme gebe, da im Gegensatz zum Nationalsozialismus die analytische Untersuchung des Sowjetkommunismus unscharf sei und ein empirisches Defizit aufweise, plante Arendt daraufhin Anfang der 1950er-Jahre eine originäre Untersuchung mit dem Titel Die marxistischen Elemente totaler Herrschaft bzw. »die totalitären Elemente des Marxismus«. »Sie wollte«, so Elisabeth Young-Bruehl, »den zentralen Unterschied zwischen Nazismus und Bolschewismus, den Unterschied zwischen Naturideologie und der Geschichtsideologie" genauer verdeutlichen. ${ }^{432}$ Ihre ursprüngliche Absicht, die philosophischen Wurzeln des sowjetischen Kommunismus aus der marxschen Theorie bzw. »der politischen Philosophie des Westens « herauszudestillieren, konnte letzten Endes nicht zum Abschluss gebracht werden, weil unter anderem ein dafür notwendiges Stipendium bei der Guggenheim Foundation nicht verlängert wurde. Allerdings verwandelte sich ihr Projekt »in einen Plan für die Grundlegung einer neuen Politikwissenschaft «. ${ }^{433}$ Sämtliche schriftlichen Arbeiten von Arendt aus den Jahren zwischen 1952 bis 1956 waren eigentlich für die »kleine Studie über Marx« gedacht, wobei das Projekt schlussendlich nicht abgeschlossen wurde. Gleichwohl entstanden aus dem eigentlich geplanten Marxismus-Buch die drei Untersuchungen Between Past and Future ${ }^{434}$, The Human Condition $^{435}$ und On Revolution ${ }^{436}$. Zudem entstand in den 1950er-Jahren unter anderem das Buch Die ungarische Revolution und der totalitäre Imperialismus ${ }^{437}$. Arendt gehörte zu den prominenten Teilnehmern der unter Vorsitz von Carl J. Friedrich am 5. März 1953 in Boston stattgefundenen Totalitarismuskonferenz. ${ }^{438}$

Nachdem Arendt in den 1950er-Jahren eine Professur am Brooklyn College in New York und eine Gastprofessur in Berkeley innehatte, trat sie Anfang der 1960er-Jahre eine Professur an der Universität von Chicago an. 1961 besuchte sie als Berichterstatterin der amerikanischen Wochenzeitschrift The New Yorker in Jerusalem den Eichmann-

431 Ebd., S. 48.

432 Young-Bruehl, Hannah Arendt S. 300.

433 Ebd., S. 385. Vgl. hierzu Hannah Arendt, Was ist Politik? Fragmente aus dem Nachlaß, München 1993.

434 Hannah Arendt, Between Past and Future. Six Exercices in Political Thought, New York 1961, erweit. Aufl. 1968 (dt. Ausg.: Zwischen Vergangenheit und Zukunft: Übungen im politischen Denken I., München/Zürich 1994). Zur Ergänzung der deutschen Ausgabe vgl. Hannah Arendt, In der Cegenwart. Übungen im politischen Denken II, München 2000 (der Band enthält Texte der politischen Philosophin, mit denen sie insbesondere auf aktuelle politische Entwicklung in der Bundesrepublik und den Vereinigten Staaten in den Jahren 1945 bis 1975 einging; zudem ist hier u. a. der 1958 erschienene Aufsatz Die Ungarische Revolution und der totalitäre Imperialismus wiederabgedruckt).

435 Dies., The Human Condition, Chicago 1958 (dt. Ausg.: Vita activa oder Vom tätigen Leben, Stuttgart 1960).

436 Dies., On Revolution, New York 1963 (dt. Ausg.: Über die Revolution, München 1963).

437 Dies., Die ungarische Revolution und der totalitäre Imperialismus, München 1958.

438 Siehe hierzu die entsprechenden Ausführungen im Einleitungskapitel. 
Prozess und löste 1963 mit der Veröffentlichung ihres Buches, ${ }^{439}$ in dem sie im Zusammenhang mit der nationalsozialistischen Judenvernichtung zum einen das Verhalten der Judenräte kritisierte und zum anderen mit Blick auf Adolf Eichmann die These von der Banalität des Bösen aufwarf, eine heftige politische und wissenschaftliche Kontroverse aus. ${ }^{440}$

Arendt nahm 1967 eine Professur an der New School for Social Research an und begann mit den Vorarbeiten für ihr philosophisches Buch The Life of the Mind. ${ }^{441}$ Nachdem sie ihre beiden wichtigsten Freunde verloren hatte - 1969 starb Karl Jaspers und 1970 Heinrich Blücher -, starb Arendt am 4. Dezember 1975 im Alter von 69 Jahren nach einem Herzinfarkt in ihrer Wohnung in New York.

\subsection{Peter de Mendelssohn}

Der britisch-deutsche Journalist und Schriftsteller Peter de Mendelssohn gehörte nach der nationalsozialistischen Machteroberung zu den "Emigranten der ersten Stunde« (Hilde Spiel). Er wurde am 1. Juni 1908 in München als Sohn eines Goldschmieds geboren. Die Mutter stammte aus Argentinien. Sein »einziger jüdischer Großvater« war ein Gelehrter von Rang und mit dem renommierten Historiker Theodor Mommsen befreundet. ${ }^{442}$ Nach dem Abitur begann er, in der Redaktion des Berliner Tageblattes mitzuarbeiten, und konnte sich mit verschiedenen literarischen Veröffentlichungen einen Namen machen. ${ }^{443}$ Am 1. April 1933 wurde de Mendelssohn mit seinem Vater in Berlin Augenzeuge der antisemitischen Boykottaktion durch die NSDAP und verließ kurz darauf - »viele unserer Freunde und Bekannten hatten sich (bereits) Mord und Totschlag und anderer Drangsalierung durch Flucht über die Grenze entzogen ${ }^{444}$ - Deutschland in Richtung »Pariser Exil« und ging über Wien 1936 noch vor dem »Anschluss Österreichs« an Deutschland nach London. Dort heiratete er die jüdisch-österreichische Schriftstellerin Hilde Spiel ${ }^{445}$ und erhielt 1941 die britische Staatsbürgerschaft. Diese

439 Eichmann in Jerusalem: A Report on the Banality of Evil, New York 1963 (dt. Ausg.: Eichmann in Jerusalem. Ein Bericht von der Banalität des Bösen, München 1964).

440 Siehe hierzu F. A. Krummacher (Hg.), Die Kontroverse. Hannah Arendt, Eichmann und die Juden, München 1964.

441 Hannah Arendt, The Life of the Mind, 2 Bde., New York 1978 (dt. Ausg.: Vom Leben des Geistes, Bd. 1: Das Denken, Bd. 2: Das Wollen, München 1979). Vgl. hierzu sowie primär zu den philosophischen Studien Arendts - mit Ausnahme von Eichmann in Jerusalem - die in den Vereinigten Staaten nach ihrer Rückkehr aus Europa 1950 angefertigten Serien von Aufzeichnungen: Hannah Arendt, Denktagebuch. 1950-1973, 2 Bde., München 2002.

442 Peter de Mendelssohn, Der Geist in der Despotie. Versuche über die Möglichkeiten des Intellektuellen in der totalitären Gesellschaft. Mit einem Vorwort von Karl Jaspers, Berlin-Grunewald 1953, S. 9.

443 Siehe hierzu Hilde Spiel, Der Erzähler Peter de Mendelssohn, in: Peter de Mendelssohn, Die Kathedrale. Ein Sommernachtmahr. Mit einem Nachwort von Hilde Spiel, Berlin 1988, S. 237-264.

444 De Mendelssohn, Der Geist in der Despotie, S. 9.

445 Hilde Spiel wurde 1911 in Wien geboren (gestorben 1990 in Wien) und wuchs im Milieu des assimilierten jüdischen Bürgertums auf. Sie emigrierte 1936 nach London. Dort arbeitete sie für verschiedene internationale Zeitungen und Zeitschriften und setzte ihre schriftstellerische Tätigkeit fort. Nach dem Krieg kehrte sie mit de Mendelssohn nach Berlin zurück und war dort als Theaterkritikerin aktiv und schrieb u. a. in der Die Neue Zeitung. Spiel zählte wie ihr Mann zu den Autoren der Zeitschrift Der Monat. Sie veröffentlichte dort insbesondere Artikel über die englische Literatur. Siehe zu Spiel 